Copyright (C) 2013 IEEE. Personal use of this material is permitted. Permission from IEEE must be obtained for all other uses, in any current or future media, including reprinting/republishing this material for advertising or promotional purposes, creating new collective works, for resale or redistribution to servers or lists, or reuse of any copyrighted component of this work in other works. 


\title{
Robust eigenstructure assignment in the computation of friends of output-nulling subspaces
}

\author{
Lorenzo Ntogramatzidis and Robert Schmid
}

\begin{abstract}
In this paper we develop a strategy for the computation of basis matrices of output-nulling subspaces, as well as of reachability and stabilisability output-nulling subspaces, with the simultaneous computation of the corresponding friend that also delivers a robust closed-loop eigenstructure. We show that the methods introduced in this paper offer considerably more robust eigenstructure assignment than the other commonly used methods employing subspace recursions.
\end{abstract}

\section{INTRODUCTION}

In this paper we introduce a new computational framework for the robust computation of friends of characteristic subspaces of linear time-invariant (LTI) systems. In the last forty years, geometric control has played a central role in the understanding of several structural properties of dynamical systems and in the solution of important control and estimation problems. The monographs [19], [2], [17], [4] provide surveys of the extensive literature in this area.

The subspaces that underpin the classic geometric theory of LTI systems are the so-called output-nulling subspaces. Two related families of subspaces, that also play a fundamental role in control and estimation problems, are the reachability and stabilisability output-nulling subspaces.

The computation of friends of output nulling spaces has been considered by many authors and the texts [2] and [4] included publicly available MATLAB ${ }^{\circledR}$ toolboxes. However, none of the methods offered in those texts have considered the problem of obtaining friends that also deliver a robust closed-loop eigenstructure that renders the closed-loop eigenvalues as insensitive to perturbations in the state matrices as possible.

In this paper we consider alternative computational methods for obtaining these subspaces via the system Rosenbrock pencil matrix [12]. An early work in this direction was made by Moore and Laub in [8], who proposed an algorithm for the computation of the largest reachability output-nulling subspace. That paper made a number of restrictive assumptions, and perhaps consequently the methods in [8] have only been given marginal attention. To the best of the authors' knowledge, the recent paper [10] was the first in the past few decades to further develop and extend the algorithms that were presented in [8] under less restrictive assumptions, and

This work was supported in part by the Australian Research Council (grant FT120100604).

L. Ntogramatzidis is with the Department of Mathematics and Statistics, Curtin University, Perth, WA, Australia 1.ntogramatzidis@curtin.edu.au

Robert Schmid is with the Department of Electrical and Electronic Engineering, The University of Melbourne, VIC, Australia. rschmid@unimelb.edu.au to adapt them to a wider class of output-nulling subspaces. In this paper we continue this investigation, and consider the problem of computing the associated friends. In Section VI we offer a comprehensive parametric formula for all the friends of the largest reachability output-nulling subspace, and in Section VII we use this parametric form to address the problem of robust eigenstructure conditioning. We propose a nonlinear unconstrained optimisation problem to find a friend that minimises the Frobenius condition number of the matrix of closed-loop eigenvectors. We consider some example systems and compare the robustness performance of the friend delivered by our method against that of the friend computed by the GA toolbox of [2], and the Linear Systems Toolkit of [4]. We observe that our method delivers dramatically improved eigenvalue insensitivity.

For the sake of simplicity of exposition, in this paper we restrict our attention to the case of distinct eigenvalues and invariant zeros.

Notation. Throughout this paper, the symbol $0_{q}$ stands for the origin of the vector space $\mathbb{R}^{q}$. The image and the kernel of matrix $A$ are denoted by $\operatorname{im} A$ and $\operatorname{ker} A$, respectively. The Moore-Penrose pseudo-inverse of $A$ is denoted by $A^{\dagger}$. Given a linear map $A: \mathscr{X} \longrightarrow \mathscr{Y}$ and a subspace $\mathscr{S}$ of $\mathscr{Y}$, the symbol $A^{-1} \mathscr{S}$ stands for the inverse image of $\mathscr{S}$ with respect to the linear map $A$. If $\mathscr{J} \subseteq \mathscr{X}$, the restriction of the map $A$ to $\mathscr{J}$ is denoted by $A \mid \mathscr{J}$. If $\mathscr{X}=\mathscr{Y}$ and $\mathscr{J}$ is $A$-invariant, the eigenvalues of $A$ restricted to $\mathscr{J}$ are denoted by $\sigma(A \mid \mathscr{J})$. If $\mathscr{J}_{1}$ and $\mathscr{J}_{2}$ are $A$-invariant subspaces and $\mathscr{J}_{1} \subseteq \mathscr{J}_{2}$, the mapping induced by $A$ on the quotient space $\mathscr{J}_{2} / \mathscr{J}_{1}$ is denoted by $A \mid \mathscr{J}_{2} / \mathscr{J}_{1}$. The symbol $\oplus$ stands for the direct sum of subspaces. Given a map $A: \mathscr{X} \longrightarrow \mathscr{X}$ and a subspace $\mathscr{B}$ of $\mathscr{X}$, we denote by $\langle A, \mathscr{B}\rangle$ the smallest $A$-invariant subspace of $\mathscr{X}$ containing $\mathscr{B}$. The symbol $i$ stands for the imaginary unit, i.e., $\mathfrak{i}=\sqrt{-1}$. The symbol $\bar{\alpha}$ denotes the complex conjugate of $\alpha \in \mathbb{C}$. Given a matrix $M$, we denote by $M_{i}$ its $i$-th row and by $M^{j}$ its $j$-th column, respectively. The normal rank of a rational matrix $M(\lambda)$ is defined as normrank $M(\lambda) \triangleq \max _{\lambda \in \mathbb{C}} \operatorname{rank} M(\lambda)$.

\section{PReliminaries}

Consider an LTI system $\Sigma$ modelled by

$$
\Sigma:\left\{\begin{array}{l}
\dot{x}(t)=A x(t)+B u(t), \quad x(0)=x_{0}, \\
y(t)=C x(t)+D u(t),
\end{array}\right.
$$

where, for all $t \geq 0, x(t) \in \mathscr{X}=\mathbb{R}^{n}$ is the state, $u(t) \in \mathscr{U}=$ $\mathbb{R}^{m}$ is the control input, $y(t) \in \mathscr{Y}=\mathbb{R}^{p}$ is the output, and $A$, $B, C$ and $D$ are appropriate dimensional constant real-valued 
matrices. Let the system $\Sigma$ described by (1) be identified by the quadruple $(A, B, C, D)$. We assume with no loss of generality that all the columns of $\left[\begin{array}{l}B \\ D\end{array}\right]$ and all the rows of $\left[\begin{array}{ll}C & D\end{array}\right]$ are linearly independent.

We define the Rosenbrock system matrix pencil as

$$
P_{\Sigma}(\lambda) \triangleq\left[\begin{array}{cc}
A-\lambda I & B \\
C & D
\end{array}\right], \quad \lambda \in \mathbb{C},
$$

[8]. Recall that the invariant zeros of $\Sigma$ are the values of $\lambda \in \mathbb{C}$ for which the rank of $P_{\Sigma}(\lambda)$ is strictly smaller than its normal rank, and the invariant zero structure is given by the zeros, multiplicity included, of the greatest common divisor of the minors of order $n+\min \{m, p\}$ of $P_{\Sigma}(\lambda)$, see [7], [1]. We denote by $\mathscr{Z}$ the set of invariant zeros of $\Sigma$.

We use the symbol $N_{\Sigma}(\lambda)$ to denote a basis matrix for the null-space of $P_{\Sigma}(\lambda)$, and we denote by $d(\lambda)$ the dimension of this null-space. Let $d \triangleq n+m-\operatorname{normrank} P_{\Sigma}(\lambda)$. Clearly $d(\lambda)=d$, unless $\lambda$ is an invariant zero of $\Sigma$ (in which case $d(\lambda)>d)$.

For any matrix $M$ with $n+m$ rows, we define $\bar{\pi}\{M\}$ and $\underline{\pi}\{M\}$ by taking the upper $n$ and lower $m$ rows of $M$, respectively.

Geometric background. Geometric objects extensively used in this paper are defined here. A controlled invariant subspace $\mathscr{V}$ of the pair $(A, B)$ is a subspace of $\mathscr{X}$ satisfying $A \mathscr{V} \subseteq \mathscr{V}+\operatorname{im} B$. An output-nulling subspace of $\Sigma=(A, B, C, D)$ is a controlled invariant subspace $\mathscr{V}$ of $\Sigma$ which satisfies $\left[\begin{array}{l}A \\ C\end{array}\right] \mathscr{V} \subseteq\left(\mathscr{V} \oplus 0_{p}\right)+\operatorname{im}\left[\begin{array}{l}B \\ D\end{array}\right]$ or, equivalently, for which two matrices $\Xi$ and $\Omega$ exist such that $\left[\begin{array}{l}A \\ C\end{array}\right] V=$ $\left[\begin{array}{l}V \\ 0\end{array}\right] \Xi+\left[\begin{array}{l}B \\ D\end{array}\right] \Omega$, where $V$ is a basis matrix of $\mathscr{V}$.

These conditions are equivalent to the existence of a matrix $F \in \mathbb{R}^{m \times n}$ such that $(A+B F) \mathscr{V} \subseteq \mathscr{V} \subseteq \operatorname{ker}(C+D F)$. Any such matrix $F$ is referred to as a friend of $\mathscr{V}$. The largest output-nulling subspace of $\Sigma$ is denoted with $\mathscr{V}^{\star}$, and represents the set of all initial states $x_{0}$ of (1) for which a control $u$ exists such that the corresponding output $y$ is identically zero. Such input function can always be implemented as a static state feedback of the form $u(t)=F x(t)$ where $F$ is a friend of $\mathscr{V}^{\star}$. The so-called largest reachability outputnulling subspace on $\mathscr{V}^{\star}$, here denoted with the symbol $\mathscr{R}^{\star}$, is the smallest $(A+B F)$-invariant subspace of $\mathscr{X}$ containing the subspace $\mathscr{V}^{\star} \cap B \operatorname{ker} D$, where $F$ is a friend of $\mathscr{V}^{\star}$. Loosely speaking, this subspace represents the states that are reachable from the origin on a state trajectory for which the output is zero, [17, Ch. 8], [9]. If $F$ is a friend of $\mathscr{V}^{\star}$, it is also a friend of $\mathscr{R}^{\star}$. The spectrum $\sigma\left(A+B F \mid \mathscr{R}^{\star}\right)$ is assignable, whereas the spectrum $\sigma\left(A+B F \mid \mathscr{V}^{\star} / \mathscr{R}^{\star}\right)$ is fixed, and its elements are the invariant zeros of $\Sigma$. Similarly, if we denote by $\mathscr{R}_{0}$ the reachability subspace from the origin, i.e., $\mathscr{R}_{0}=\langle A, \operatorname{im} B\rangle=\operatorname{im}\left[\begin{array}{llll}B & A B & \ldots & A^{n-1} B\end{array}\right]$, the spectrum $\sigma\left(A+B F \mid \mathscr{V}^{\star}+\mathscr{R}_{0} / \mathscr{V}^{\star}\right)$ is assignable, whereas the spectrum $\sigma\left(A+B F \mid \mathscr{X} / \mathscr{V}^{\star}+\mathscr{R}_{0}\right)$ is fixed.

\section{Computation OF $\mathscr{R}^{\star}$}

The first aim of this section is to provide a generalisation of a series of results, see e.g. [8, Proposition 4], on the relationship existing between the largest reachability outputnulling subspace with the null-space of the Rosenbrock system matrix pencil.

Given a set of $h$ self-conjugate complex numbers $\mathscr{L}=$ $\left\{\lambda_{1}, \ldots, \lambda_{h}\right\}$ containing exactly $s$ complex conjugate pairs, we say that $\mathscr{L}$ is $s$-conformably ordered if the first $2 s$ values of $\mathscr{L}$ are complex while the remaining are real, and for all odd $k \leq 2 s$ we have $\lambda_{k+1}=\bar{\lambda}_{k}$. For example, the sets $\mathscr{L}_{1}=\{1+\mathfrak{i}, 1-\mathfrak{i}, 3,-4\}, \mathscr{L}_{2}=\{10 \mathfrak{i},-10 \mathfrak{i}, 2+2 \mathfrak{i}, 2-2 \mathfrak{i}, 7\}$ and $\mathscr{L}_{3}=\{3,-1\}$ are respectively $1-, 2$ - and 0 -conformably ordered.

The following theorem constitutes the main result of this section. It provides a method to construct a basis for $\mathscr{R}^{\star}$ and simultaneously a friend $F$ that assigns the eigenstructure of the closed-loop restricted to $\mathscr{R}^{\star}$. This theorem also identifies the degree of freedom that can be exploited in order to develop the theory for the robust eigenstructure assignment established in Section VII.

Theorem 3.1: Let $r=\operatorname{dim} \mathscr{R}^{\star}$. Let $\mathscr{L}=\left\{\lambda_{1}, \ldots, \lambda_{r}\right\}$ be $s$-conformably ordered and such that $\mathscr{L} \cap \mathscr{Z}=\emptyset$. Let $K \triangleq$ $\operatorname{diag}\left\{K_{1}, \ldots, K_{r}\right\}$, where $K_{i} \in \mathbb{C}^{d}$ (recall that $d=n+m-$ normrank $P_{\Sigma}$ ) for each $i \in\{1, \ldots, 2 s\}$, and for all odd $i \leq 2 s$, we have $\bar{K}_{i}=K_{i+1}$, whereas $K_{i} \in \mathbb{R}^{d}$ for $i \in\{2 s+1, \ldots, r\}$. Let $M_{K}$ be an $(n+m) \times r$ complex matrix given by

$$
M_{K} \triangleq\left[N_{\Sigma}\left(\lambda_{1}\right)\left|N_{\Sigma}\left(\lambda_{2}\right)\right| \ldots \mid N_{\Sigma}\left(\lambda_{r}\right)\right] K
$$

and let for all $j \in\{1, \ldots, r\}$

$$
m_{K, j} \triangleq \begin{cases}\mathfrak{R e}\left\{M_{K}^{j}\right\} & \text { if } j \leq 2 s \text { is odd } \\ \mathfrak{I m}\left\{M_{K}^{j}\right\} & \text { if } j \leq 2 s \text { is even } \\ M_{K}^{j} & \text { if } j>2 s\end{cases}
$$

Finally, let

$$
\begin{aligned}
& X_{K} \triangleq \bar{\pi}\left\{\left[\begin{array}{llll}
m_{K, 1} & m_{K, 2} & \ldots & m_{K, r}
\end{array}\right]\right\}, \\
& Y_{K} \triangleq \underline{\pi}\left\{\left[\begin{array}{llll}
m_{K, 1} & m_{K, 2} & \ldots & m_{K, r}
\end{array}\right]\right\} .
\end{aligned}
$$

For almost every choice of the parameter matrix $K=$ $\operatorname{diag}\left\{K_{1}, \ldots, K_{r}\right\}$, the rank of $X_{K}$ is equal to $r$. Moreover, for all $K$ is such that $\operatorname{rank} X_{K}=r$, there holds $\mathscr{R}^{\star}=\operatorname{im} X_{K}$, and the set of all friends of $\mathscr{R}^{\star}$ such that $\sigma\left(A+B F \mid \mathscr{R}^{\star}\right)=\mathscr{L}$ is parameterised as

$$
F_{K}=Y_{K} X_{K}^{\dagger}
$$

We now present a simple numerical case, which will be used as a running example in this paper.

Example 3.1: Consider a quadruple $(A, B, C, D)$ where

$$
\begin{aligned}
& A=\left[\begin{array}{lll}
0 & 0 & 0 \\
0 & 3 & 0 \\
0 & 0 & 0
\end{array}\right], \quad B=\left[\begin{array}{ll}
1 & 0 \\
2 & 0 \\
0 & 3
\end{array}\right], \\
& C=\left[\begin{array}{lll}
0 & 0 & 0
\end{array}\right], \quad D=\left[\begin{array}{ll}
0 & 4
\end{array}\right] .
\end{aligned}
$$


The only invariant zero of this system is $z=0$. It is easy to verify that $\mathscr{R}^{\star}$ is spanned by the first two canonical basis vectors of $\mathbb{R}^{3}$. Hence, $r=\operatorname{dim} \mathscr{R}^{\star}=2$. Let us choose for example $\mathscr{L}=\left\{\lambda_{1}, \lambda_{2}\right\}=\{-2,-4\}$. Basis matrices for $\operatorname{ker} P_{\Sigma}(-2)$ and $\operatorname{ker} P_{\Sigma}(-4)$ are given respectively by $N_{\Sigma}(-2)=\left[\begin{array}{lll}5 & 4 & 0\end{array} \mid-\right.$

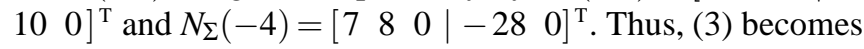

$$
M_{K}=\left[\begin{array}{c|c}
5 & 7 \\
4 & 8 \\
0 & 0 \\
\hline-10 & -28 \\
0 & 0
\end{array}\right] K, \quad \text { where } \quad K=\left[\begin{array}{cc}
k_{1} & 0 \\
0 & k_{2}
\end{array}\right]
$$

By choosing $k_{1}=k_{2}=1$, we find $X_{K}=\left[\begin{array}{ll}5 & 7 \\ 4 & 8 \\ 0 & 0\end{array}\right]$ and $Y_{K}=$ $\left[\begin{array}{cc}-10 & -28 \\ 0 & 0\end{array}\right]$. Thus, as expected $\operatorname{im} X_{K}=\mathscr{R}^{\star}$, and $F_{K}=Y_{K} X_{K}^{\dagger}=$ $\left[\begin{array}{ccr}8 / 3 & -35 / 6 & 0 \\ 0 & 0 & 0\end{array}\right]$ is a friend of $\mathscr{R}^{\star}$. Indeed, it can be immediately verified that $\left(A+B F_{K}\right) \mathscr{R}^{\star} \subseteq \mathscr{R}^{\star} \subseteq \operatorname{ker}\left(C+D F_{K}\right)$. Moreover, with this choice of $F$ the eigenvalue of $\left(A+B F_{K}\right)$ restricted to $\mathscr{R}^{\star}$ are indeed $\{-2,-4\}$.

Remark 3.1: The fact that Theorem 3.1 assumes the knowledge of the dimension of $\mathscr{R}^{\star}$ does not constitute a limitation in the calculation of a basis for $\mathscr{R}^{\star}$ and of the corresponding friend. Indeed, one can progressively compute the submatrices $N_{\Sigma}\left(\lambda_{1}\right), N_{\Sigma}\left(\lambda_{2}\right)$, and so on, for different values of $\lambda_{i}$, until a value $\lambda_{k}$ is found for which $\operatorname{rank}\left[N_{\Sigma}\left(\lambda_{1}\right)|\ldots| N_{\Sigma}\left(\lambda_{k-1}\right)\right]=\operatorname{rank}\left[N_{\Sigma}\left(\lambda_{1}\right)|\ldots| N_{\Sigma}\left(\lambda_{k}\right)\right]$. The dimension of $\mathscr{R}^{\star}$ equals the rank of $\left[N_{\Sigma}\left(\lambda_{1}\right)|\ldots| N_{\Sigma}\left(\lambda_{k}\right)\right]$. Theorem 3.1 guarantees that almost any choice of the $\lambda_{i}$ will lead to the determination of a basis for $\mathscr{R}^{\star}$.

\section{Assigning the OUter Eigenstructure of $\mathscr{R}^{\star}$}

In the previous section, we showed how to construct a friend $F$ of the subspace $\mathscr{R}^{\star}$ that arbitrarily assigns all the eigenvalues of the closed-loop restricted to $\mathscr{R}^{\star}$. However, we also know that the spectrum induced by the map $A+B F$ on the quotient space $\mathscr{R}_{0}+\mathscr{R}^{\star} / \mathscr{R}^{\star}=\mathscr{R}_{0} / \mathscr{R}^{\star}$ (where $\mathscr{R}_{0} \triangleq$ $\langle A, \operatorname{im} B\rangle$ is the classic (Kalman) reachable subspace from the origin) is assignable using a friend $F$. The following result shows how Theorem 3.1 can be adapted to this case.

Theorem 4.1: Let $r=\operatorname{dim} \mathscr{R}^{\star}$ and $r_{0}=\operatorname{dim} \mathscr{R}_{0}$. Let $\mathscr{L}_{\text {in }}=\left\{\lambda_{1}, \ldots, \lambda_{r}\right\}$ be $s_{\text {in }}$-conformably ordered with elements all different from the invariant zeros, and let $\mathscr{L}_{\text {out }}=$ $\left\{\mu_{1}, \ldots, \mu_{r_{0}-r}\right\}$ be $s_{\text {out }}$-conformably ordered with elements all different from the uncontrollable eigenvalues of the pair $(A, B)$ with $\mathscr{L}_{\text {in }} \cap \mathscr{L}_{\text {out }}=\emptyset$. Let $K \triangleq \operatorname{diag}\left\{k_{1}, \ldots, k_{r}\right\}$ be defined as in Theorem 3.1 for $\mathscr{L}=\mathscr{L}_{\text {in }}$. Moreover, let $K^{\prime} \triangleq$ $\operatorname{diag}\left\{k_{1}^{\prime}, \ldots, k_{r_{0}-r}^{\prime}\right\}$, where $k_{i}^{\prime} \in \mathbb{C}^{m}$ for each $i \in\left\{1, \ldots, 2 s_{\text {out }}\right\}$, and for all odd $i \leq 2 s_{\text {out }}$, we have $\bar{k}_{i}^{\prime}=k_{i+1}^{\prime}$, whereas $k_{i}^{\prime} \in \mathbb{R}^{m}$ for $i \in\left\{2 s_{\text {out }}+1, \ldots, r_{0}-r\right\}$. Let $M_{K, K^{\prime}}$ be an $(n+m) \times r_{0}$ complex matrix given by

$$
\begin{aligned}
& M_{K, K^{\prime}}=\left[N_{\Sigma}\left(\lambda_{1}\right)\right. \ldots N_{\Sigma}\left(\lambda_{r}\right) \\
&\left.S_{\Sigma}\left(\mu_{1}\right) \ldots S_{\Sigma}\left(\mu_{r_{0}-r}\right)\right] \operatorname{diag}\left\{K, K^{\prime}\right\}
\end{aligned}
$$

where $S_{\Sigma}(\mu)$ represents a basis matrix for $\operatorname{ker}\left[\begin{array}{lll}A-\mu I_{n} & B\end{array}\right]$, and let $m_{K, K^{\prime}, j}$ be defined as

$$
m_{K, K^{\prime}, j}= \begin{cases}\mathfrak{R e}\left\{M_{K, K^{\prime}}^{j}\right\} & \text { if } j \leq 2 s_{\text {in }} \text { odd or } r<j<r+2 s_{\text {out }} \\ & \text { is such that } r+j \text { is odd } \\ \mathfrak{I m}\left\{M_{K, K^{\prime}}^{j}\right\} & \text { if } j \leq 2 s_{\text {in }} \text { even or } r<j<r+2 s_{\text {out }} \\ & \text { is such that } r+j \text { is even } \\ M_{K, K^{\prime}}^{j} & \text { if } j \in\left\{2 s_{\text {in }}+1, \ldots, r\right\} \\ & \cup\left\{r+2 s_{0}+1, \ldots, r_{0}\right\}\end{cases}
$$

Finally, let $X_{K, K^{\prime}}=\bar{\pi}\left\{\left[\begin{array}{lll}m_{K, K^{\prime}, 1} & \ldots & m_{K, K^{\prime}, r_{0}}\end{array}\right]\right\}$ and $Y_{K, K^{\prime}}=$ $\underline{\boldsymbol{\pi}}\left\{\left[\begin{array}{lll}m_{K, K^{\prime}, 1} & \ldots & m_{K, K^{\prime}, r_{0}}\end{array}\right]\right\}$. For almost every choice of $K$ and $K^{\prime}$ we have $\operatorname{rank} \bar{\pi}\left\{\left[\begin{array}{lll}m_{K, K^{\prime}, 1} & \ldots & m_{K, K^{\prime}, r}\end{array}\right]\right\}=r$ and $\operatorname{rank} X_{K, K^{\prime}}=r_{0}$. Moreover, the set of all friends of $\mathscr{R}^{\star}$ such that $\sigma\left(A+B F \mid \mathscr{R}^{\star}\right)=\mathscr{L}_{\text {in }}$ and $\sigma\left(A+B F \mid \mathscr{R}_{0} / \mathscr{R}^{\star}\right)=\mathscr{L}_{\text {out }}$ is parameterised in $K$ and $K^{\prime}$ as

$$
F_{K, K^{\prime}}=Y_{K, K^{\prime}} X_{K, K^{\prime}}^{\dagger}
$$

where $K$ and $K^{\prime}$ are such that $\operatorname{rank} X_{K, K^{\prime}}=r_{0}$ (and therefore, for such $K$ and $K^{\prime}$, the matrix $X_{K, K^{\prime}}$ represents a basis for $\mathscr{R}_{0}$ adapted to $\left.\mathscr{R}^{\star}\right)$.

A proof of this result can be carried out following the same arguments of the proof of Theorem 3.1, and is omitted.

Example 4.1: Consider the system in Example 3.1. Since the pair $(A, B)$ is reachable, we can compute a friend $F$ of $\mathscr{R}^{\star}$ by assigning a further eigenvalue of $(A+B F)$ which corresponds to the map induced by $A+B F$ on the quotient space $\mathscr{R}_{0} / \mathscr{R}^{\star}=\mathscr{X} / \mathscr{R}^{\star}$. Assume that $\mathscr{L}_{\text {in }}=\mathscr{L}=$ $\{-2,-4\}$ and $\mathscr{L}_{\text {out }}=\{-6\}$. We have already computed

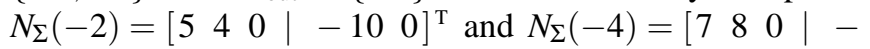
$280^{\mathrm{T}}$. A basis matrix of $\operatorname{ker}\left[A-(-6) I_{3} \quad B\right]$ is given by $S_{\Sigma}(-6)=\left[\begin{array}{cccccc}3 & 4 & 0 & \text { | } & -18 & 0 \\ 0 & 0 & -1 & \mid & 0 & 2\end{array}\right]^{\mathrm{T}}$. Thus, choosing for example $K=\operatorname{diag}\left\{k_{1}, k_{2}\right\}=\operatorname{diag}\{1,1\}$ and $K^{\prime}=k_{1}^{\prime}=\left[\begin{array}{l}0 \\ 1\end{array}\right]$ we get $X_{K, K^{\prime}}=\operatorname{diag}\left\{\left[\begin{array}{ll}5 & 7 \\ 4 & 8\end{array}\right],-1\right\}$ and $Y_{K, K^{\prime}}=\left[\begin{array}{ccc}-10 & -28 & 0 \\ 0 & 0 & 2\end{array}\right]$. Then, with $F_{K, K^{\prime}}=Y_{K, K^{\prime}} X_{K, K^{\prime}}^{\dagger}=Y_{K, K^{\prime}} X_{K, K^{\prime}}^{-1}=\left[\begin{array}{ccc}8 / 3 & -35 / 6 & 0 \\ 0 & 0 & -2\end{array}\right]$, we find $\left(A+B F_{K, K^{\prime}}\right) \mathscr{R}^{\star} \subseteq \mathscr{R}^{\star} \subseteq \operatorname{ker}\left(C+D F_{K, K^{\prime}}\right)$. The eigenvalues of $\left(A+B F_{K, K^{\prime}} \mid \mathscr{R}^{\star}\right)$ are $\{-2,-4\}$, while the eigenvalue induced by $\left(A+B F_{K, K^{\prime}}\right)$ on $\mathscr{R}_{0} / \mathscr{R}^{\star}$ is -6 .

\section{Computation of $\mathscr{V}^{\star}$}

The following theorem adapts Theorem 3.1 to the case of the subspace $\mathscr{V}^{\star}$.

Theorem 5.1: Let $r=\operatorname{dim} \mathscr{R}^{\star}$. Let all the invariant zeros of the system be distinct. Let $\mathscr{Z}=\left\{z_{1}, z_{2}, \ldots, z_{t}\right\}$ be the $s_{z^{-}}$ conformably ordered set of invariant zeros of $\Sigma$. Let $\mathscr{L}=$ $\left\{\lambda_{1}, \ldots, \lambda_{r}\right\}$ be $s$-conformably ordered such that $\mathscr{L} \cap \mathscr{Z}=$ $\emptyset$. Let the column vectors $m_{K, j}$, for all $j \in\{1, \ldots, r\}$, be constructed as in (4). Let $H \triangleq \operatorname{diag}\left\{h_{1}, \ldots, h_{t}\right\}$, where $h_{i} \in$ $\mathbb{C}^{\operatorname{dimker} P_{\Sigma}\left(z_{i}\right)}$ for each $i \in\left\{1, \ldots, 2 s_{z}\right\}$, and for all odd $i \leq 2 s_{z}$, we have $\bar{h}_{i}=h_{i+1}$, whereas $h_{i} \in \mathbb{R}^{\operatorname{dim} \operatorname{ker} P_{\Sigma}\left(z_{i}\right)}$ for $i \in\left\{2 s_{t}+\right.$ $1, \ldots, r\}$. Let $M_{H}$ be a complex matrix given by

$$
M_{H}=\left[N_{\Sigma}\left(z_{1}\right)\left|N_{\Sigma}\left(z_{2}\right)\right| \ldots \mid N_{\Sigma}\left(z_{t}\right)\right] H
$$


and let for all $j \in\{1, \ldots, t\}$

$$
m_{H, j}= \begin{cases}\mathfrak{R e}\left\{M_{H}^{j}\right\} & \text { if } j \leq 2 s_{z} \text { is odd } \\ \mathfrak{I m}\left\{M_{H}^{j}\right\} & \text { if } j \leq 2 s_{z} \text { is even } \\ M_{H}^{j} & \text { if } j>2 s_{z}\end{cases}
$$

Finally, let

$$
\begin{aligned}
X_{K, H} & =\bar{\pi}\left\{\left[\begin{array}{llllll}
m_{K, 1} & \ldots & m_{K, r} & m_{H, 1} & \ldots & m_{H, t}
\end{array}\right]\right\}, \\
Y_{K, H} & =\underline{\pi}\left\{\left[\begin{array}{llllll}
m_{K, 1} & \ldots & m_{K, r} & m_{H, 1} & \ldots & m_{H, t}
\end{array}\right]\right\} .
\end{aligned}
$$

For almost every choice of the parameter matrices $K=\operatorname{diag}\left\{k_{1}, \ldots, k_{r}\right\}$ and $H=\operatorname{diag}\left\{h_{1}, \ldots, h_{t}\right\}$ we have $\operatorname{rank} X_{K, H}=r+t$. Moreover, the set of all friends of $\mathscr{V}^{\star}$ such that $\sigma\left(A+B F \mid \mathscr{V}^{\star}\right)=\mathscr{L} \cup \mathscr{Z}$ is parameterised in $K$ and $H$ as

$$
F_{K, H}=Y_{K, H} X_{K, H}^{\dagger}
$$

where $K, H$ are such that $\operatorname{rank} X_{K}=r+t$.

The proof can be carried out along the same lines of the proof of Theorem 3.1, taking into account [8, Proposition 5]. Notice that the same procedure can be used for the computation of $\mathscr{V}_{g}^{\star}$, by considering only the minimum-phase invariant zeros in the set $\mathscr{Z}$.

Example 5.1: Consider again the system in Example 3.1. We want to compute a basis for $\mathscr{V}^{\star}$ and a friend of $\mathscr{V}^{\star}$ such that $\sigma\left(A+B F \mid \mathscr{R}^{\star}\right)=\{-2,-4\}$. Since this system has an invariant zero at the origin, this task can be accomplished with a friend such that $\sigma\left(A+B F \mid \mathscr{V}^{\star}\right)=\{-2,-4,0\}$. We have

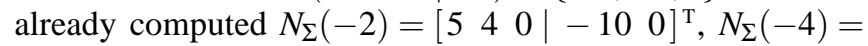
$\left[\begin{array}{lll|ll}7 & 8 & 0 & -28 & 0\end{array}\right]^{\mathrm{T}}$. A basis matrix for $\operatorname{ker}\left[\begin{array}{lll}A-0 \cdot I_{n} & B\end{array}\right]$ is

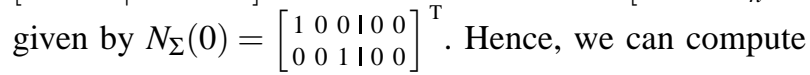

$$
M_{K}=\left[\begin{array}{c|c|cc}
5 & 7 & 1 & 0 \\
4 & 8 & 0 & 0 \\
0 & 0 & 0 & 1 \\
\hline-10 & -28 & 0 & 0 \\
0 & 0 & 0 & 0
\end{array}\right]\left[\begin{array}{c|c|c}
k_{1} & 0 & 0 \\
\hline 0 & k_{2} & 0 \\
\hline 0 & 0 & k_{3}^{\prime} \\
0 & 0 & k_{3}^{\prime \prime}
\end{array}\right]
$$

Choosing for example $k_{1}=k_{2}=1, k_{3}^{\prime}=0$ and $k_{3}^{\prime \prime}=1$, we find $X_{K}=\left[\begin{array}{lll}5 & 7 & 0 \\ 4 & 8 & 0 \\ 0 & 0 & 1\end{array}\right], Y_{K}=\left[\begin{array}{ccc}-10 & -28 & 0 \\ 0 & 0 & 0\end{array}\right]$, which yield $F_{K}=Y_{K} X_{K}^{\dagger}=$ $\left[\begin{array}{ccc}8 / 3 & -35 / 6 & 0 \\ 0 & 0 & 0\end{array}\right]$. Clearly, $\left(A+B F_{K}\right) \mathscr{V}^{\star} \subseteq \mathscr{V}^{\star} \subseteq \operatorname{ker}(C+$ $\left.D F_{K}\right)$ and $\sigma\left(A+B F_{K} \mid \mathscr{V}^{\star}\right)=\sigma(A+B F)=\{0,-2,-4\}$ as required.

\section{COMPUTATION OF FRIENDS WITH OUTER SPECTRAL ASSIGNMENT}

We now show that it is always possible to parameterise all the friends that assign the internal and external eigenstructure of $\mathscr{V}^{\star}$ by means of a formula

$$
F_{K}=Y_{K} X_{K}^{-1}
$$

i.e., where this time $X_{K}$ is square and invertible (for almost all choices of the parameter matrix $K$ ). This step is crucial in the robust computation of friends. For the sake of simplicity of exposition, we assume that all the internal/external eigenvalues to be assigned, as well as all the invariant zeros and uncontrollable modes of the pair $(A, B)$ are real and distinct. The complex conjugate case follows straightforwardly by applying the result in Theorem 4.1.

Theorem 6.1: [PARAMETERISATION OF FRIENDS OF $\mathscr{V} \star$ WITH INNER-OUTER SPECTRUM ASSIGNMENT]

Let $r=\operatorname{dim} \mathscr{R}^{\star}$. Let $\mathscr{L}_{\text {in }}=\left\{\lambda_{1}, \ldots, \lambda_{r}\right\}$ be $s_{\text {in }}$-conformably ordered. Let $\mathscr{Z}=\left\{z_{1}, \ldots, z_{t}\right\}$ be the set of invariant zeros. Let $\mathscr{L}_{\text {out }}=\left\{\mu_{1}, \ldots, \mu_{q}\right\}$ be $s_{\text {out }}$-conformably ordered, where $q=\operatorname{dim}\left(\mathscr{R}_{0}+\mathscr{V}^{\star}\right)-\operatorname{dim}\left(\mathscr{V}^{\star}\right)$. Finally, let $\mathscr{G}=\left\{g_{1}, \ldots, g_{\eta}\right\}$ represent the uncontrollable eigenvalues of $(A, B)$, where $\eta=$ $n-\operatorname{dim} \mathscr{R}_{0}$. We assume without loss of generality that $\mathscr{L}_{\text {in }} \cap$ $\mathscr{Z}=\emptyset$ and that $\mathscr{L}_{\text {out }} \cap \mathscr{G}=\emptyset$. Define

$$
\begin{aligned}
& M_{K}=\left[\begin{array}{llllll}
N_{\Sigma}\left(\lambda_{1}\right) & \ldots & N_{\Sigma}\left(\lambda_{r}\right) \mid N_{\Sigma}\left(z_{1}\right) & \ldots & N_{\Sigma}\left(z_{t}\right)
\end{array}\right. \\
& \left.S_{\Sigma}\left(\mu_{1}\right) \ldots S_{\Sigma}\left(\mu_{q}\right) \mid S_{\Sigma}\left(g_{1}\right) \ldots S_{\Sigma}\left(g_{\eta}\right)\right] K,
\end{aligned}
$$

where $K=\operatorname{diag}\left\{K_{\lambda}, K_{z}, K_{\mu}, K_{g}\right\}$, and

- $K_{\lambda}=\operatorname{diag}\left\{k_{1}^{\lambda}, \ldots, k_{r}^{\lambda}\right\}$, with $k_{i}^{\lambda} \in \mathbb{R}^{d}$, and where $d=$ $\operatorname{dim} \operatorname{ker} P_{\Sigma}(\lambda)$ when $\lambda$ is not an invariant zero;

- $K_{z}=\operatorname{diag}\left\{k_{1}^{z}, \ldots, k_{t}^{z}\right\}$, with $k_{i}^{z} \in \mathbb{R}^{d_{z}}$, and $d_{z}=$ $\operatorname{dim} \operatorname{ker} P_{\Sigma}(z)$ when $z \in \mathscr{Z}$;

- $K_{\mu}=\operatorname{diag}\left\{k_{1}^{\mu}, \ldots, k_{q}^{\mu}\right\}$, with $k_{i}^{\mu} \in \mathbb{R}^{m}$, since $m=$ $\operatorname{dim} \operatorname{ker} S_{\Sigma}(\lambda)$ when $\lambda$ is not an uncontrollable eigenvalue of the pair $(A, B)$;

- $K_{g}=\operatorname{diag}\left\{k_{1}^{g}, \ldots, k_{\eta}^{g}\right\}$, with $k_{i}^{g} \in \mathbb{R}^{m_{g}}$, and where $m_{g}=$ $\operatorname{dim} \operatorname{ker} S_{\Sigma}(g)$ when $g$ is an uncontrollable eigenvalue of the pair $(A, B)$.

Finally, define

$$
X_{K}=\bar{\pi}\left\{M_{K}\right\} \in \mathbb{R}^{n \times n} \quad \text { and } \quad Y_{K}=\underline{\pi}\left\{M_{K}\right\} \in \mathbb{R}^{m \times n} .
$$

For almost every choice of $K$, the matrix $X_{K}$ is invertible, and the set of all friends of $\mathscr{V}^{\star}$ such that $\sigma\left(A+B F \mid \mathscr{R}^{\star}\right)=\mathscr{L}_{\text {in }}$, $\sigma\left(A+B F \mid \mathscr{V}^{\star} / \mathscr{R}^{\star}\right)=\mathscr{Z}$ and $\sigma\left(A+B F \mid \mathscr{R}_{0}+\mathscr{V}^{\star} / \mathscr{V}^{\star}\right)=$ $\mathscr{L}_{\text {out }}-$ and therefore obviously $\sigma\left(A+B F \mid \mathscr{X} / \mathscr{R}_{0}+\mathscr{V}^{\star}\right)=$ $\mathscr{G}$ - is parameterised in $K$ as

$$
F_{K}=Y_{K} X_{K}^{-1}
$$

where $K$ is such that $X_{K}$ is invertible. Moreover, for such $K$ the first $r$ columns of $X_{K}$ are a basis for $\mathscr{R}^{\star}$, the first $r+t$ columns of $X_{K}$ are a basis for $\mathscr{V}^{\star}$ and the first $r+t+q$ are a basis for $\mathscr{V}^{\star}+\mathscr{R}_{0}$.

\section{THE COMPUTATION OF FRIENDS FOR ROBUST EIGENSTRUCTURE}

In this section we consider the problem of obtaining friends of output-nulling subspaces that also yield a robust closed loop eigenstructure. For any square matrix $M$, it was shown in [18] that the sensitivity of the eigenvalue $\lambda_{i}$ to perturbations in $M$ can be measured by the condition number

$$
c_{i}=\frac{\left\|y_{i}\right\|\left\|v_{i}\right\|}{\left|y_{i}^{\mathrm{T}} v_{i}\right|},
$$

where $v_{i}$ and $y_{i}$ are the right and left eigenvectors of $\lambda_{i}$, respectively. We use $c_{\infty} \triangleq \max _{i} c_{i}$ to denote the worst-case eigenvalue sensitivity. Furthermore, in [6] the sensitivity of the eigenvalues is linked to measures of the conditioning of 
the matrix $X$ of eigenvectors of $M$, in terms of the Euclidean and Frobenius norms:

$$
c_{\infty} \leq \kappa_{2}(X) \leq \kappa_{\mathrm{FRO}}(X)
$$

where $\kappa_{2}(X) \triangleq\|X\|_{2} \cdot\left\|X^{-1}\right\|_{2}$ and $\kappa_{\mathrm{FRO}}(X) \triangleq\|X\|_{\mathrm{FRO}}$. $\left\|X^{-1}\right\|_{\text {FRO }}$ are the condition numbers of $X$ with respect to the 2 -norm and the Frobenius norm respectively.

For pairs $(A, B)$, the problem of finding a gain matrix $F$ that assigns a certain set of desired eigenvalues $\mathscr{L}$ to the matrix $A+B F$ and also minimises these condition numbers is known as the robust pole placement problem, and has an extensive literature. Notable contributions include [6], [16] and the recent paper [11]. For quadruples $(A, B, C, D)$ we introduce the robust friend computation problem, which involves obtaining a friend of an output-nulling subspace that assigns a certain desired set of internal and external closed-loop eigenvalues, and also a robust closed-loop eigenstructure. Despite several authors explicitly acknowledged the need for a development of robust techniques within the geometric framework [8], [5], to date there have been no results on this problem.

We now consider how the parameterisation of the friends given in Theorem 3.1 can be adapted for this problem. According to Theorem 6.1, if all eigenvalues to be assigned, invariant zeros and uncontrollable eigenvalues are real, we can express $X$ - where from now on we omit the dependence upon the parameter matrix $K-$ as

$$
X=\bar{\pi}\left\{\left[\begin{array}{lll}
G_{1} & \ldots & G_{V}
\end{array}\right]\right\} \operatorname{diag}\left\{k_{1}, \ldots, k_{V}\right\}
$$

where $G_{i}$ is a basis of the kernel of either the Rosenbrock matrix $N_{\Sigma}\left(\lambda_{i}\right)$ (or $N_{\Sigma}\left(z_{i}\right)$ ), or of the matrix pencil $S_{\Sigma}\left(\lambda_{i}\right)$ (or $\left.S_{\Sigma}\left(g_{i}\right)\right)$. Moreover, we define the column vector

$$
\xi \triangleq\left[\begin{array}{c}
k_{1} \\
k_{2} \\
\vdots \\
k_{v}
\end{array}\right],
$$

and we denote by $l_{i}$ the number of components of $k_{i}$, so that $\xi$ has $r d+t d_{z}+q m+\eta m_{g}=l_{1}+\ldots+l_{v}$ components. With these definitions, we can now exploit a crucial result in [3], which says that minimising $\kappa_{\mathrm{FRO}}(X)$ is equivalent to minimising the alternative objective function

$$
f_{2}(\xi) \triangleq\|X(\xi)\|_{\mathrm{FRO}}^{2}+\left\|X^{-1}(\xi)\right\|_{\mathrm{FRO}}^{2}
$$

In order to use gradient descent methods to obtain local minima of this function, the first and second derivatives of $\|X\|_{\text {FRO }}$ and $\left\|X^{-1}\right\|_{\text {FRO }}$ are employed. The full computation of the derivatives is outlined in [13]. Then, using either Newton's method or quasi-Newton one, we achieve a solution to the problem of minimising $\kappa_{\mathrm{FRO}}(X)$, thereby providing good robustness for the eigenvalues of the closed-loop system.

\section{AN ILLUSTRATIVE EXAMPLE}

Consider the following quadruple

$$
\begin{aligned}
& A=\left[\begin{array}{cccccccc}
0 & 6 & -4 & 0 & 0 & 0 & 0 & 0 \\
-2 & 0 & 0 & 0 & 0 & 7 & 0 & 0 \\
0 & -9 & -9 & -10 & 8 & 0 & 0 & 6 \\
2 & 0 & 0 & 0 & 0 & -2 & -4 & 0 \\
0 & 0 & 0 & 0 & 0 & 0 & -3 & 6 \\
9 & 0 & 1 & 0 & -1 & 0 & 0 & 0 \\
0 & 0 & -8 & 0 & 0 & 0 & -3 & 0 \\
-3 & 0 & 0 & -10 & -3 & 0 & 8 & 0
\end{array}\right], \quad B=\left[\begin{array}{ccc}
0 & 0 & 0 \\
9 & 2 & 5 \\
0 & 0 & 0 \\
0 & -5 & 0 \\
0 & 0 & 0 \\
0 & 0 & -6 \\
0 & 0 & 0 \\
0 & 0 & -3
\end{array}\right], \\
& C=\left[\begin{array}{lllllll}
-7 & 0 & -4 & 0 & 0 & 0 & 0
\end{array}\right], \quad D=\left[\begin{array}{lll}
0 & 0 & 0
\end{array}\right] \text {. }
\end{aligned}
$$

In this example, we have $\mathscr{V}^{\star}=\mathscr{R}^{\star}, \operatorname{dim} \mathscr{R}^{\star}=6$, and the pair $(A, B)$ is reachable, so that $\mathscr{R}_{0}=\mathscr{X}$. This system has no invariant zeros. We want to find a friend $F$ of $\mathscr{R}^{\star}$ such that $\mathscr{L}_{\text {in }}=\{-1,-2,-3,-4,-5,-6\}$ and $\mathscr{L}_{\text {out }}=\{-7,-8\}$. Using the routine effesta.m in the toolbox $G A$ [2], we find that a friend that accomplishes this task is given by

$F_{1}=\left[\begin{array}{cccccccc}-0.0648 & -3.3046 & -0.1467 & 0.0853 & 0.4753 & -0.7881 & -0.0953 & -1.0966 \\ -1.1223 & -1.5083 & 0.9430 & 0.8138 & 0.2027 & -0.3425 & -1.0706 & -0.1055 \\ -0.3199 & 3.9952 & 0.7620 & -0.5444 & -0.8419 & 0.1628 & 0.5686 & 2.0574\end{array}\right]$

Using the routine atea.m in the toolbox Linear Systems Toolkit [4], we find that an alternative friend that achieves the same goal, and is given by

$F_{2}=\left[\begin{array}{cccccccc}3.3163 & -1.3615 & -1.1872 & -0.4456 & 0.8412 & -0.7835 & -0.0651 & -0.9233 \\ -2.9733 & -2.7000 & 1.6130 & 1.0320 & -0.1698 & -0.3660 & -0.9051 & -0.3408 \\ -5.3155 & 0.4780 & 2.8057 & -0.1256 & -2.2519 & 0.0498 & 1.4428 & 1.0926\end{array}\right]$

Using the gradient iterative method developed in this paper, we obtain

$F_{3}=\left[\begin{array}{cccccccc}-0.1495 & -0.8175 & -0.3581 & 2.0241 & -0.4644 & -0.7285 & -0.5987 & -1.8265 \\ -1.0727 & -3.0008 & 1.0185 & -0.3810 & 0.8063 & -0.3769 & -0.7796 & 0.3626 \\ -0.1783 & -0.3409 & 0.8712 & -4.0825 & 0.9965 & 0.0659 & 1.3905 & 3.4818\end{array}\right]$

To compare these friends of $\mathscr{V}^{\star}$, we consider several performance measures. Computing the conditioning measure $c_{\infty}$ in (16) arising from each friend, we observe that $c_{\infty}\left(F_{1}\right)=624$ while $c_{\infty}\left(F_{2}\right)=7144, c_{\infty}\left(F_{3}\right)=61.7$, indicating that our method gives reduced eigenvalue sensitivity, by one and two orders of magnitude, respectively. We may also compare norms of these gain matrices. We obtain $\left\|F_{1}\right\|_{2}=5.20$, $\left\|F_{2}\right\|_{2}=8.18$, while $\left\|F_{3}\right\|_{2}=6.42$, indicating that our method used somewhat higher gain that of effesta.m, but less than atea.m for this example. By further considering a weighted robustness and gain minimisation problem,

$$
\min _{\xi}\left\{\alpha f_{2}(\xi)+(1-\alpha)\|F\|_{F}^{2}\right\}
$$

we can sacrifice some robustness in exchange for reduced gain. Choosing $\alpha=0.001$, we were able to obtain a matrix

$F_{4}=\left[\begin{array}{cccccccc}-0.0914 & -1.8620 & -0.8391 & 0.8569 & 0.0904 & -0.7584 & -0.2856 & -1.4403 \\ -1.1672 & -2.3583 & 1.3484 & 0.3826 & 0.4125 & -0.3600 & -0.9600 & 0.0853 \\ -0.5807 & 1.5595 & 1.9179 & -1.7291 & -0.2776 & 0.1125 & 0.8822 & 2.5786\end{array}\right]$

yielding eigenvalue sensitivity $c_{\infty}\left(F_{4}\right)=67.63$ and gain $\left\|F_{4}\right\|_{2}=4.94$, and thus offering improvement over $F_{1}$ on both criteria.

Another performance consideration is the accuracy of the pole placement achieved by each method. We use the measure

$$
\Delta(F) \triangleq \max \left\{\left|\operatorname{eig}_{i}(A+B F)-\lambda_{i}\right|: \lambda_{i} \in \mathscr{L}\right\}
$$


which represents the largest absolute value difference between each eigenvalue of $A+B F$, and the corresponding $\lambda_{i}$ in $\mathscr{L}$. We obtained $\Delta\left(F_{1}\right)=1.16 \times 10^{-12}, \Delta\left(F_{2}\right)=2.34 \times 10^{-11}$ and $\Delta\left(F_{3}\right)=3.20 \times 10^{-14}, \Delta\left(F_{4}\right)=3.74 \times 10^{-14}$, indicating that our method achieved more accurate pole placement, again by some orders of magnitude.

\section{CONCLUDING REMARKS}

This paper provides new procedures for the computation of bases matrices for the fundamental output-nulling subspaces $\mathscr{R}^{\star}, \mathscr{V}^{\star}$ and $\mathscr{V}_{g}^{\star}$ as well as of their corresponding friends which robustly assigns their free internal and external eigenstructure. We compared the method introduced in this paper against the two most widely known and used MATLAB ${ }^{\circledR}$ toolboxes for the computation of such subspaces to show the dramatic improvement of the eigenvalue sensitivity.

An important direction of future research is the exploitation of the results presented in this paper to the problems of designing linear state feedback control laws that yield an non-overshooting and non-undershooting step response for an LTI systems as studied in [14] and [15], based on the computation of the Rosenbrock matrix.

\section{REFERENCES}

[1] H. Aling and J.M. Schumacher. A nine-fold canonical decomposition for linear systems. International Journal of Control, 39(4):779-805, 1984.

[2] G. Basile and G. Marro. Controlled and Conditioned Invariants in Linear System Theory. Prentice Hall, Englewood Cliffs, New Jersey, 1992.

[3] R. Byers and S. G. Nash, Approaches to robust pole assignment, International Journal of Control, vol. 49, pp. 97-117, 1989.

[4] B. Chen, Z. Lin, Y. Shamash, Linear systems theory: a structural decomposition approach. Birkhäuser, Boston, 2004.

[5] D. Chu and V. Mehrmann, Disturbance decoupling for linear timeinvariant systems: a matrix pencil approach, IEEE Transactions on Automatic Control, vol. AC-46, no. 5, pp. 802-808, 2001.

[6] J. Kautsky, J. N.K. Nichols and P. Van Dooren, Robust Pole Assignment in Linear State Feedback, International Journal of Control, vol. 41 , pp. $1129-1155,1985$.

[7] A.G.J. MacFarlane and N. Karcanias. Poles and zeros of linear multivariable systems: a survey of the algebraic, geometric and complex variable theory. International Journal of Control, pages 33-74, 1976.

[8] B.C. Moore, and A.J. Laub, Computation of Supremal $(A, B)$-Invariant and Controllability Subspaces, IEEE Transactions on Automatic Control, vol. AC-23, no. 5, pp. 783-792, 1978.

[9] L. Ntogramatzidis, "Self-bounded output-nulling subspaces for non strictly proper systems and their application to the disturbance decoupling problem". IEEE Transactions on Automatic Control, vol. 53, no. 1, pp. 423-428, 2008.

[10] L. Ntogramatzidis, R. Schmid and A. Ferrante, "On the computation of reachability, stabilisability and output-nulling subspaces using the Rosenbrock system matrix". In Proceedings of the $2^{\text {nd }}$ Australian Control Conference, Sydney, 2012.

[11] M. Ait Rami, S.E. Faiz, A. Benzaouia, and F. Tadeo, Robust Exact Pole Placement via an LMI-Based Algorithm, IEEE Transactions on Automatic Control, Vol. 54(2) pp. 394-398, 2009.

[12] H. H. Rosenbrock, State-Space and Multivariable Theory. New York: Wiley, 1970

[13] R. Schmid, T. Nguyen and A. Pandey, Optimal Pole placement with Moore's algorithm, in Proceedings 1st IEEE Australian Control Conference (AUCC 2011), Melbourne, Australia, 2011.

[14] R. Schmid, and L. Ntogramatzidis, A unified method for the design of nonovershooting linear multivariable state-feedback tracking controllers. Automatica, 46: 312-321, 2010.

[15] R. Schmid, and L. Ntogramatzidis, "The design of nonovershooting and nonundershooting multivariable state feedback tracking controllers". Systems \& Control Letters, 61:714-722, 2012.
[16] A. L. Tits and Y. Yang, Globally Convergent Algorithms for Robust Pole Assignment by State Feedback, IEEE Transactions on Automatic Control., vol. 41(10), pp. 1432-1452, 1996.

[17] H. Trentelman, A. Stoorvogel, and M. Hautus, Control theory for linear systems, ser. Communications and Control Engineering. Great Britain: Springer, 2001.

[18] J. H. Wilkinson, The Algebraic Eigenvalue Problem, Oxford University Press, 1965.

[19] W.M. Wonham. Linear Multivariable Control: A Geometric Approach. Springer-Verlag, 3 edition, 1985. 